\title{
Cohesive Crack Model to Reflect Local Chemical Action at Grain and Its Boundaries in Polycrystalline Metals*
}

\begin{abstract}
by Yuichi Shintaku**, Mayu Muramatsu***, Shinsuke Takase****, Seiichiro Tsutsumi***** and Kenjiro Terada******
The objective of this study is to develop a new cohesive zone model to assess the residual strength of the structure subject to local chemical action of hydrogen. A damage variable representing material degradations is introduced to a cohesive zone model by use of the thermodynamics approach. Our model, in which the decrease of the energy release rate caused by hydrogen is considered, enables us to evaluate the hydrogen embrittlement. Some representative numerical examples are presented to demonstrate the performance of the proposed model in simulating fracture processes accelerated by the hydrogen concentration.
\end{abstract}

Key Words: Hydrogen Embrittlement, Crack Propagation, Crystal Plasticity, Grain Boundary Diffusion,

Cohesive Zone Model, Thermodynamics-based Damage

\section{Introduction}

The hydrogen embrittlement, which is more generally called environment-assisted cracking, remains incompletely understood from the mechanical point of view. It becomes more and more important to understand the hydrogen embrittlement for the safety of our lives because the hydrogen might be an alternative energy to oil. The hydrogen embrittlement especially occurs in high-strength steel, which is used as a prestressed concrete steel and high-strength bolt, under long-term use. In the oil industry, it often becomes a problem since the crude oil includes the hydrogen sulfide ${ }^{1)}$. If energy necessary for life shifts to hydrogen energy from oil energy, the hydrogen embrittlement will be more serious problem ${ }^{2}$.

In fracture processes due to hydrogen embrittlement in polcrystalline aggregates, the hydrogen tends to attack grain boundaries rather than the inside of crystal grains, since the diffusion velocity of hydrogen at the former is faster than that in the latter ${ }^{3)}$. Under the assumption that the hydrogen concentration decreases the surface energy ${ }^{4}$, we propose a new cohesive zone model to simulate trans- and inter-granular crack propagations promoted by hydrogen concentration. Such a dependency of the energy release rate on the hydrogen concentration is represented on some theoretical basis. Also, to represent the effect of voids

\footnotetext{
${ }^{*}$ Received: 2014.11.28

** Student Member, Department of Civil and Environmental Engineering, Tohoku University

${ }^{* * *}$ Member, Graduate School of Environmental Studies, Tohoku University

*** Member, Department of Civil and Environmental Engineering, Tohoku University

${ }^{* * * * *}$ Member, Jointing and Welding Research Institute, Osaka University

${ }^{* * * * * * *}$ Member, International Research Institute of Disaster Science, Tohoku University
}

caused by plastic deformation, a damage variable is incorporated into an existing cohesive zone model within the framework of thermodynamics. In addition to the cohesive zone model, a crystal plasticity model is employed to represent the grain boundaries. A numerical example shows a double-cantilever beam test influenced by hydrogen embrittlement, which demonstrates the ability of the proposed model.

\section{Cohesive zone model to reflect local chemical action}

2.1 Cohesive zone model based on atomic potential and damage variable representing material degradation

The cohesive zone model represents the separation process of a continuum body into two or more parts, with controlling the release of the internal stress. At the atomic scale, the material separation is characterized by the relation between the atomic force and the distance between the crack surfaces, which is generally given by the universal binding energy relation $(\mathrm{UBER})^{5)}$. Since the atomic separation takes place in the brittle fracture, Rice and Wang ${ }^{6}$ employ UBER in order to models the process of the brittle fracture. On the other hands, it is required to consider the material degradation caused by the nucleation, growth and coalescence of micro crack and void in the ductile, fatigue and creep fractures ${ }^{1}$. According to these two conceptions, we propose a new cohesive zone model which is based on UBER. The damage variable $D$ is introduced to express the material degradation. The cohesive potential $\Psi$ is given as

$$
\Psi=(1-D) G_{\mathrm{c}}\left[1-\left(1+\frac{\delta}{\delta_{\mathrm{c}}}\right) \exp \left(-\frac{\delta}{\delta_{\mathrm{c}}}\right)\right],
$$

where $G_{\mathrm{c}}$ is the critical energy release rate, $\delta$ is the effective opening displacement, which is defined as 


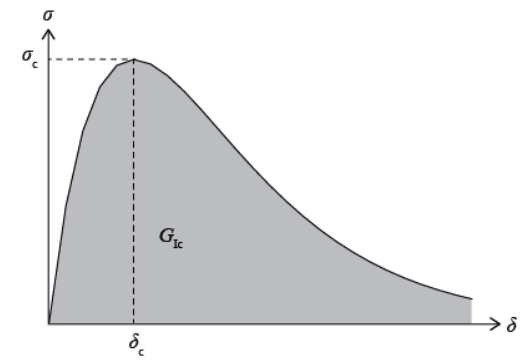

Fig. 2 Relationship between cohesive traction and separation distance

$$
\delta=\sqrt{\delta_{\mathrm{n}}^{2}+\beta \delta_{\mathrm{t}}^{2}} .
$$

Here, $\delta_{\mathrm{n}}$ is the normal component of the opening displacement to the crack surface, $\delta_{\mathrm{t}}$ is the tangential component, and $\beta$ is the weighting coefficient to control the effect of the tangential opening displacement. The $\delta_{\mathrm{c}}$ is the critical value of $\delta$ when the cohesive stress reaches a maximum as shown in Fig. 1. The critical energy release rate $G_{\mathrm{c}}$ is given as

$$
G_{\mathrm{c}}=\int_{0}^{\infty} \sigma \mathrm{d} \delta,
$$

where $\sigma$ is the effective cohesive traction, which is defined as

$$
\sigma=\sqrt{\sigma_{\mathrm{n}}^{2}+\beta^{-1}\left|\sigma_{\mathrm{t}}\right|^{2}}
$$

The cohesive tractions in the normal and tangential directions, $\sigma_{\mathrm{n}}$ and $\sigma_{\mathrm{t}}$, are given as the derivatives of the potential with respect to the normal and tangential displacements $\delta_{\mathrm{n}}$ and $\delta_{\mathrm{t}}$, respectively, as

$$
\begin{aligned}
& \sigma_{\mathrm{n}}=\frac{\partial \Psi}{\partial \delta_{\mathrm{n}}}=(1-D) \frac{G_{\mathrm{c}}}{\delta_{\mathrm{c}}}\left(\frac{\delta_{\mathrm{n}}}{\delta_{\mathrm{c}}}\right) \exp \left(-\frac{\delta}{\delta_{\mathrm{c}}}\right), \\
& \sigma_{\mathrm{t}}=\frac{\partial \Psi}{\partial \delta_{\mathrm{t}}}=\beta(1-D) \frac{G_{\mathrm{c}}}{\delta_{\mathrm{c}}}\left(\frac{\delta_{\mathrm{t}}}{\delta_{\mathrm{c}}}\right) \exp \left(-\frac{\delta}{\delta_{\mathrm{c}}}\right) .
\end{aligned}
$$

Until $\delta$ becomes $\delta_{\mathrm{c}}, \sigma$ increases as shown in Fig. 2 and then it decreases asymptotically to zero.

The thermodynamic force $Y$ can also be obtained as the derivative of the cohesive potential with respect to $D$

$$
Y=\frac{\partial \Psi}{\partial D}=G_{\mathrm{c}}\left[1-\left(1+\frac{\delta}{\delta_{\mathrm{c}}}\right) \exp \left(-\frac{\delta}{\delta_{\mathrm{c}}}\right)\right],
$$

which represents the energy dissipation due to damage progress. The evolution law of $D$ is given by the following equations, which was originally presented by Bouvard et al. ${ }^{7)}$.

$$
\begin{array}{cc}
\dot{D}=B(1-D)^{m}\left\langle\sqrt{Y}-\sqrt{Y_{\text {th }}}\right\rangle^{n}\left\|\frac{\dot{\delta}}{\delta_{\mathrm{c}}}\right\| \quad \text { (if } \dot{Y} \geq 0 \text { ), } \\
\dot{D}=0 & \text { (otherwise), }
\end{array}
$$

where $B, m$ and $n$ are the material parameters, and $Y_{\text {th }}$ is the threshold value. In this model, the damage occurs after the $Y$ exceeds $Y_{\text {th }}$ and the damage accumulates due to the increase of crack opening under loading $(\dot{Y} \geq 0)$. Figure 2 shows a schematic of the response of $\sigma$ with $\delta$ under cyclic loading. The cohesive traction goes back to the origin under unloading condition.

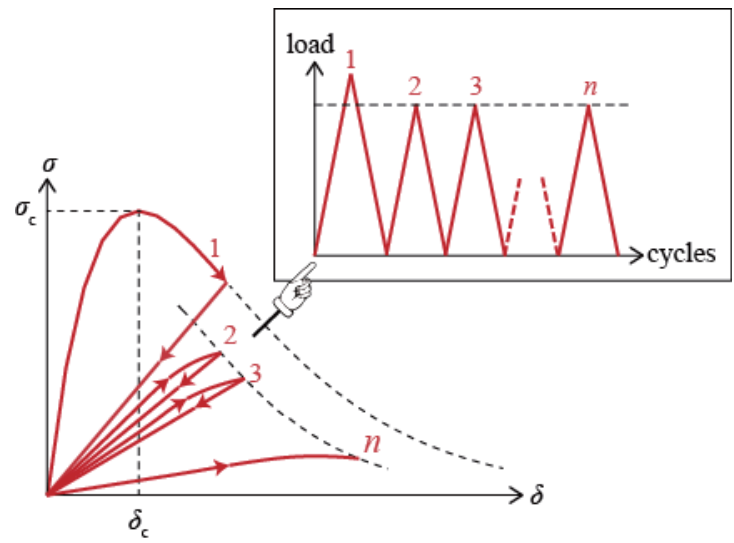

Fig. 1 Schematic of the response of stress

with the effective opening displacement under cyclic loading

During the subsequent reloading, it goes on to the different path from that under unloading condition owing to the damage variable. The damage variable increases only under the loading, while it keeps constant under the unloading.

\subsection{Decrease in energy release rate due to hydrogen}

According to Griffith-Orowan-Irwin theory ${ }^{1)}, G_{\mathrm{c}}$ is devided into the surface energy $\omega_{\mathrm{s}}$ and the plastic work $\omega_{\mathrm{p}}$.

$$
G_{\mathrm{c}}=2\left(\omega_{\mathrm{s}}+\omega_{\mathrm{p}}\right) .
$$

Rimoli and Ortiz ${ }^{8)}$ approximate the change of $\omega_{\mathrm{s}}$ on the based on the investigation by Jiang et al. ${ }^{4}$.

$$
\omega_{\mathrm{s}}=\left(1-1.0467 \theta+0.1687 \theta^{2}\right) \omega_{\mathrm{s} 0},
$$

where $\theta=c / c_{\mathrm{s}}, c$ is the surface concentration, $c_{\mathrm{s}}$ is the saturation value of $c$ and $\omega_{\mathrm{s} 0}$ is the surface energy when $c=0$. Jokl et al. ${ }^{9)}$ demonstrate $\omega_{\mathrm{s}}$ and $\omega_{\mathrm{p}}$ interact with each other; the increase of $\omega_{\mathrm{s}}$ involves the rapid increase of $\omega_{\mathrm{p}}$. Therefore, the change of $\omega_{\mathrm{p}}$ is expressed by the same form as eq. (11). As the result, the change of $G_{\mathrm{c}}$ is given as

$$
G_{\mathrm{c}}=\left(1-1.0467 \theta+0.1687 \theta^{2}\right) G_{\mathrm{c} 0},
$$

where $G_{\mathrm{c} 0}$ is the surface energy when $c=0$.

Although the change of $\omega_{\mathrm{s}}$ may take the different process due to the type of the crystal structure of metal, the decrease of $\omega_{\mathrm{s}}$ according to the increase of $c$ is common to $\mathrm{BCC}$ and $\mathrm{FCC}$ crystals $^{4)}$. From this reason, we assumed the process of FCC is qualitatively similar to the one of BCC in this study. Actually, we did not employ eq. (11) but eq. (12), in which the value of $G_{\mathrm{c} 0}$ for FCC is used.

\section{Crystal plasticity model ${ }^{10}$}

The total deformation gradient is decomposed into the two parts, i.e., the elastic deformation due to distortion of crystal lattices and the plastic deformation due to crystallographic slip. 


$$
\boldsymbol{F}=\boldsymbol{F}^{\mathrm{e}} \boldsymbol{F}^{\mathrm{p}},
$$

where $\boldsymbol{F}^{\mathrm{e}}$ is the elastic deformation gradient and $\boldsymbol{F}^{\mathrm{p}}$ is the plastic deformation gradient, respectively. The velocity gradient is expressed as the additive decomposition as follows:

$$
\boldsymbol{l}:=\dot{\boldsymbol{F}} \boldsymbol{F}^{-1}=\boldsymbol{l}^{\mathrm{e}}+\boldsymbol{l}^{\mathrm{p}},
$$

where the velocity gradients due to elastic deformation $l^{\mathfrak{e}}$ and crystallographic slip $\boldsymbol{l}^{\mathrm{p}}$, which are respectively been defined as

$$
\left\{\begin{array}{c}
\boldsymbol{l}^{\mathrm{e}}:=\dot{\boldsymbol{F}}^{\mathrm{e}} \boldsymbol{F}^{\mathrm{e}-1} \\
\boldsymbol{l}^{\mathrm{p}}:=\boldsymbol{F}^{\mathrm{e}} \dot{\boldsymbol{F}}^{\mathrm{p}} \boldsymbol{F}^{\mathrm{p}-1} \boldsymbol{F}^{\mathrm{e}-1} .
\end{array}\right.
$$

The flow rule for a crystal plasticity model is given as

$$
\boldsymbol{l}^{\mathrm{p}}=\sum_{\alpha=1}^{N}\left(\boldsymbol{s}^{*(\alpha)} \otimes \boldsymbol{m}^{*(\alpha)}\right) \dot{\gamma}^{(\alpha)}
$$

where $N$ is the number of slip systems of crystal lattice of a metal, $\dot{\gamma}^{(\alpha)}$ is the slip rate in slip system $\alpha$, and $\boldsymbol{s}^{*(\alpha)}$ and $\boldsymbol{m}^{*(\alpha)}$ are defined as

$$
\left\{\begin{array}{c}
\boldsymbol{s}^{*(\alpha)}=\boldsymbol{F}^{\mathrm{e}} \boldsymbol{F}^{\mathrm{p}} \boldsymbol{s}_{0}^{(\alpha)} \\
\boldsymbol{m}^{*(\alpha)}=\boldsymbol{m}_{0}^{(\alpha)} \boldsymbol{F}^{\mathrm{p}-1} \boldsymbol{F}^{\mathrm{e}-1},
\end{array}\right.
$$

where $\boldsymbol{s}_{0}{ }^{(\alpha)}$ is the unit vector that defines the slip direction and $\boldsymbol{m}_{0}{ }^{(\alpha)}$ is the unit vector that defines the normal direction of the slip surfaces of system $\alpha$. The evolution law of $\dot{\gamma}^{(\alpha)}$ is given as the following equation, which was proposed by Asaro et al. ${ }^{10)}$

$$
\dot{\gamma}^{(\alpha)}=\dot{a}\left|\frac{\tau^{(\alpha)}}{g^{(\alpha)}}\right|^{n^{\gamma}} \operatorname{sign}\left(\frac{\tau^{(\alpha)}}{g^{(\alpha)}}\right),
$$

where $\dot{a}$ is the reference slip rate, $g^{(\alpha)}$ is the slip resistance of slip system $\alpha$ and $n^{\mathrm{V}}$ is a parameter that controls the strain rate dependency. The evolution equation of $g^{(\alpha)}$, is give as

$$
\dot{g}^{(\alpha)}:=\sum_{\beta=1}^{n} h_{\alpha \beta} \dot{\gamma}^{(\beta)},
$$

where $h_{\alpha \beta}$ represents the hardening moduli. The hardening function is defined as

$$
\left\{\begin{array}{c}
h_{\alpha \alpha}=h_{0} \sec h^{2}\left|\frac{h_{0} \gamma}{\tau_{s}-\tau_{0}}\right| \\
h_{\alpha \beta}=q_{\alpha \beta} h_{\alpha \alpha},
\end{array}\right.
$$

where $h_{\alpha \alpha}$ and $h_{\alpha \beta}$ are the self-hardening and latent hardening moduli, respectively and $q_{\alpha \beta}$ is the ratio of the latter to the former. Also, $h_{0}, \tau_{0}$ and $\tau_{\mathrm{s}}$ are the initial hardening modulus, the initial critical resolved shear stress and the stage-I (saturation) stress, respectively.

\section{Hydrogen diffusion analysis in polycrystalline metals}

Hydrogen generates due to chemical reaction on the surface of metal in the hydrogen compound, sea and water. The hydrogen penetrates into the surface and diffuses mainly at the grain boundary. The hydrogen concentration $c$ is obtained by Fick's

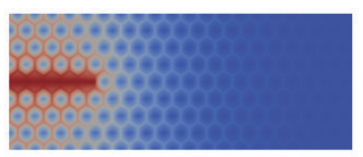

(a) $400 \mathrm{~s}$

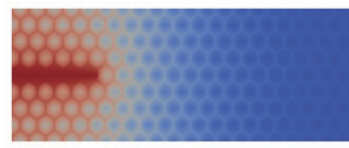

(b) $800 \mathrm{~s}$

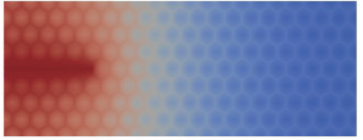

(c) $1600 \mathrm{~s}$

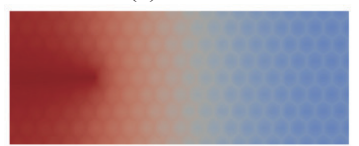

(d) $3200 \mathrm{~s}$

\section{0}

$$
\theta=c / c_{\mathrm{s}}
$$

Fig. 3 Temporal distributions of hydrogen concentration

Table 1 Material parameters for crystallographic slip ${ }^{11)}$

\begin{tabular}{|c|c|c|}
\hline$h_{0}=220$ & $\tau_{\mathrm{s}}=300[\mathrm{MPa}]$ & $\tau_{0}=93[\mathrm{MPa}]$ \\
\hline$q=1$ & $\dot{a}=0.001$ & $n^{\mathrm{V}}=30$ \\
\hline
\end{tabular}

second law as

$$
\frac{\partial c}{\partial t}+\nabla \cdot \mathbf{j}=0
$$

where $\mathbf{j}$ is the flux of the hydrogen. It is given by the following equation in Fick's first law

$$
\mathbf{j}=-K \nabla c
$$

where $K$ is the diffusion coefficient. By substituting eq. (22) into eq. (21), the diffusion equation is given as

$$
\frac{\partial c}{\partial t}=\nabla \cdot(K \nabla c) .
$$

\section{Results and discussion}

We perform a numerical analysis for the crack propagation influenced by hydrogen embrittlement in a double-cantilever-type specimen. The specimen has a spatial dimension of $1.0 \times 0.4 \times 0.025 \mathrm{~mm}$ and is given a sharp initial crack whose size is $0.25 \mathrm{~mm}$. The specimen is assumed to consist of 160 crystal grains that have the same diameter of $0.1 \mathrm{~mm}$. Also, the shape of the grain is assumed be a hexagonal prism. Then, the following two steps are taken sequentially in the numerical analysis:

(i) diffusion analysis of hydrogen concentration, and

(ii) stress analysis to realize the crack propagation.

That is, prior to the stress analysis for the specimen, the parameters for the proposed cohesive law are updated to reflect the hydrogen concentration evaluated in advance by the diffusion analysis.

In the diffusion analysis, the hydrogen is assumed to penetrate into the initial crack surface and start to diffuse in the specimen. The diffusion coefficients near the grain boundary is given as $K_{\mathrm{gb}}$ $\left.=2.53 \times 10^{-9} \mathrm{~m}^{2} / \mathrm{s}^{7}\right)$ and it inside the grain is assumed $K_{\text {in }}=$ $2.53 \times 10^{-13} \mathrm{~m}^{2} / \mathrm{s} \quad\left(K_{\mathrm{in}}=K_{\mathrm{gb}} \times 10^{4}\right)$. Under this assumption, the boundary condition $\theta=1$ is given on the initial crack surface. 


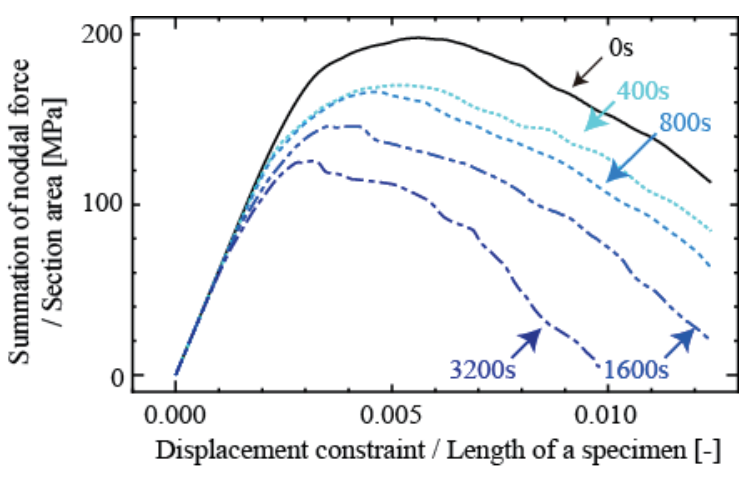

Fig. 4 Apparent response depending on the different hydrogen concentration states

Figure 3 shows the distribution of hydrogen concentration at 400 , 800,1600 and 3200s. The hydrogen initially diffuses at grain boundary at $400 \mathrm{~s}$, and then it penetrates into the grain at $3200 \mathrm{~s}$.

Afterward, five cases of the stress analysis are conducted for the different distributions of hydrogen concentration at 0,400 , 800,1600 and 3200s. The forced displacement is imposed on the upper and lower edge of specimen and the symmetry condition is applies on $x-z$ plane. The material parameters of the proposed model are set at $G_{\mathrm{c} 0}=502 \mathrm{~kJ} / \mathrm{m}, \delta_{\mathrm{c}}=2 \mu \mathrm{m}, \beta=0.7, B=180, m=$ $1.5, n=0.01$ and $Y_{\text {th }}=0.0$. Other material parameters are given in Table 1 and the elastic modulus $\boldsymbol{E}$ is given as $E_{11}=142300[\mathrm{MPa}]$, $E_{12}=124100[\mathrm{MPa}]$ and $E_{44}=95240[\mathrm{MPa}]$. These values are quoted from the literature ${ }^{11)}$. The overall response of these results is shown in Fig. 4. Here, we have defined the apparent stress as the areal average of the nodal forces at the upper edge and the apparent strain as the ratio of the displacement at the upper edge divided by the initial length along $z$ axis of the specimen. As can be seen from the figure, the apparent strength is decreased with increase of hydrogen concentration. Figure 5 shows five different profiles of the crack growths. The crack propagates inside the grain at $0 \mathrm{~s}$. The interface between grains segregates at $400 \mathrm{~s}$, and then the crack propagates inside again. Since the hydrogen diffuses at the grain boundary at 400s as shown in Fig. 3 and the hydrogen concentration inside the grain is not enough to bring into the transgranular cracking, which leads crack propagation along the grain boundary.

\section{Conclusions}

We proposed a new cohesive zone model with thermodynamics-based damage to account for the effect of hydrogen concentration. The proposed model enables us to simulate the crack propagation due to hydrogen embrittlement. It is demonstrated with the numerical example that, according to the amount of hydrogen concentration, the apparent strength is decreased and different fracture profiles are obtained.

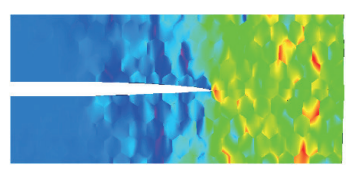

(a) $0 \mathrm{~s}$

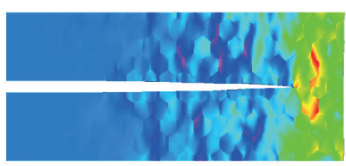

(c) $800 \mathrm{~s}$

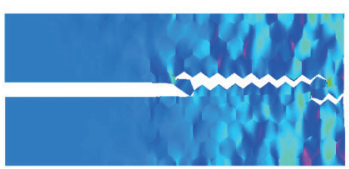

(e) $3200 \mathrm{~s}$ (b) $400 \mathrm{~s}$

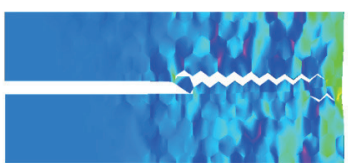

(d) $1600 \mathrm{~s}$

$-300$

[MPa]

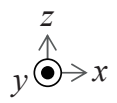

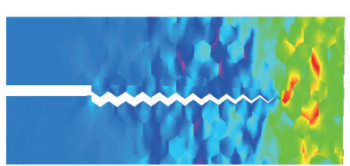

Fig. 5 Crack propagation with stress distribution at final step

\section{Acknowledgements}

This work was supported by Grant-in-Aid for JSPS Fellows Grant Number $26 \cdot 8761$.

\section{Reference}

1) T. Anderso: Fracture mechanics: Fundamentals and Applications, Boca CRC Press, Boca Raton.

2) M. Yukitaka and H. Matsunaga: The effect of hydrogen on fatigue properties of steels used for fuel cell system, Int. J. Fatigue, 28(2006), 1509-1520

3) J. C. Fisher: Calculation of diffusion penetration curves for surface and grain boundary diffusion, J. Appl. Phys. 22(1951), 74-77

4) D. E. Jiang and E. A. Carter: First principles assessment of ideal fracture energies deformation in crystalline solids, Acta. Metall., 28(1980), 1479-1488.

5) J. H. Rose and J. R. Smith: Universal binding energy curves for metals and bimetallic interfaces, Phys. Rev. Lett., 47(1981), 675-678.

6) J. Rice and J. Wang: Embrittlement of interfaces by solute segregation, Mater. Sci. Eng. A102(1989), 23-40.

7) J. L. Bourvard, J. L. Chaboche, F. Feyel and F. Gallenrneau: A cohesive zone model for fatigue and creep-fatigue crack growth in single crystal superallys, Int. J. Fatigue, 31(2009), 868-879.

8) J. J. Rimoli and M. Ortiz: A three dimentional multiscale model of intergranular hydrogen-assisted cracking, Philos. Mag., 90(2010), 2939-2963.

9) M. L. Jokl, V. Viek, and C. J. NcMachon Jr: Material rate dependence and localized deformation in crystalline solids, Acta. Mettall., 28(1980), 1479-1488.

10) J. R. Asaro: Crstal plasticity. J. Appl. Mech., 50(1983), 921-934.

11) Y. Shintaku et al.: An analysis of fatigue crack propagation in pollycrystalline metals using cohesive zone model with damage variable, Trans. Japan Soc. Comput. Eng. Sci., 2014(2014), 20140014 (in Japanese). 